\title{
Caracterización del fenómeno de «cola larga» en los portales de boca a boca electrónico
}

\author{
María Olmedilla Fernández \\ Assistant professor de la SKEMA Business School \\ mariaolmedilla@hotmail.com \\ Sergio Luis Toral Marín \\ Catedrático de universidad de la \\ Escuela Técnica Superior de Ingeniería de la Universidad de Sevilla \\ storal@us.es

\begin{abstract}
María del Rocío Martínez-Torres
Catedrática de universidad en la Facultad de Ciencias Económicas y Empresariales de la Universidad de Sevilla
\end{abstract}

rmtorres@us.es

\section{Extracto}

El comercio online y los sistemas de recomendación tienen un efecto sobre la demanda de distintos tipos de productos. El objetivo del artículo es probar si internet promueve los productos más populares o super-hit, los productos menos populares o nicho, o ambos, así como analizar cuantitativamente la coexistencia de los efectos super-hit y de "cola larga». Analizando la curva de distribución de las acciones realizadas por los consumidores en internet sobre 28 categorías de producto, se proponen dos métodos: el método de ajuste de la ley de potencia de la distribución del número de productos (factor de la oferta) por número de comentarios online (factor de la demanda) y el método del codo demarcado por el ajuste de la ley de potencia para probar matemáticamente la presencia de ambos fenómenos. Los datos se extrajeron con un crawler programado con Python y con la librería de código abierto Scrapy. Los hallazgos revelan que el boca a boca electrónico promueve estos fenómenos según las diferentes categorías de productos, así como su coexistencia. Entre las implicaciones gerenciales destacan las nuevas perspectivas sobre los mercados potenciales que pueden abrirse por la expansión de la cola.

Palabras clave: boca a boca electrónico (electronic word-of-mouth [eWOM]); contenido generado por el usuario; "cola larga»; distribución de la ley de potencia; método del codo.

Fecha de entrada: 28-06-2019 / Fecha de revisión: 10-07-2019 / Fecha de aceptación: 15-07-2019

Cómo citar: Olmedilla Fernández, M. a , Toral Marín, S. L. y Martínez-Torres, M. ${ }^{a}$ R. (2019). Caracterización del fenómeno de "cola larga» en los portales de boca a boca electrónico. Tecnología, Ciencia y Educación, 14, 97-125. 


\title{
Characterization of the long tail in eWOM websites
}

\author{
María Olmedilla Fernández \\ Sergio Luis Toral Marín \\ María del Rocío Martínez-Torres
}

\begin{abstract}
Online commerce and recommender systems influence the demand for different products. The objective of this paper is to prove if the internet promote super-hits products, niche products or both, and to analyse the coexistence of super-hit effect and long tail phenomenon using a quantitative perspective. Through the analysis of the distribution curve of the actions developed by consumers in the internet in 28 different product categories, the power-law distribution of the number of products (supply factor) through the number of online reviews (demand factor), and the elbow criterion over the case of a power law distribution are proposed to mathematically prove the presence of both phenomena. Data were capture with a crawler designed using Python and an open source web crawler framework called Scrapy. Findings reveal that eWOM promotes both the super-hit and the long tail phenomenon depending on the different product categories, and that they can coexist. As a main managerial implication, the study provides new insights about potential markets that can be open as a result of the tail expansion.
\end{abstract}

Keywords: electronic word-of-mouth (eWOM); user generated content; long tail; power law fitting; elbow criterion. 


\section{Sumario}

1. Introducción

2. Enfoque teórico y marco conceptual

2.1. Efecto super-hit versus fenómeno de "cola larga»

2.2. Boca a boca electrónico

2.3. Ajuste de las distribuciones de la ley de potencia online

3. Motivación de la investigación y preguntas de investigación

4. Metodología de investigación

4.1. Caso de estudio y recolección de datos

4.2. Técnicas cuantitativas

4.2.1. Ajuste de la ley de potencia a los datos recopilados

4.2.2. Encontrar el $x_{\min }$ óptimo según el método del codo

5. Resultados

6. Discusión e implicaciones

6.1. Discusión

6.2. Implicaciones para la gestión

6.3. Limitaciones de la investigación

6.4. Trabajo futuro

Referencias bibliográficas

Nota: este trabajo se enmarca dentro de la Convocatoria de Ayudas a Proyectos de l+D+i 2017 de la Fundación Hergar (categoría: Investigación aplicada y tecnológica en Ciencias Sociales, Jurídicas y Humanidades). 


\section{Introducción}

El efecto de internet y el aumento de la disponibilidad de información del consumidor redefinen un cambio en la distribución de las ventas online de los productos sobre los que se escriben comentarios, haciendo que productos que no son populares, también llamados «productos nicho», representen una parte importante de las ventas generales en los mercados online (Gu, Tang y Whinston, 2013). Dicha transformación en la distribución de las ventas del producto es lo que Anderson (2004) denominó «cola larga», cuyo fenómeno se puede explicar gracias a las diferentes tecnologías de búsqueda o sistemas de recomendación existentes en la actualidad, que conducen a la redistribución de la demanda, tanto de los productos nicho como de los productos más populares o super-hit.

La influencia de los sistemas de recomendación parece ser contradictoria al promover ambos tipos de productos (nicho y populares). En este sentido, estudios previos abren una brecha entre dos posturas diferentes. Por una parte, investigaciones a favor de la idea de la "cola larga» sostienen que estimular la oferta de productos accesibles a través de canales online fortalecerá las ventas de productos nicho (Elberse, 2008). Sin embargo, otras investigaciones abogan por la venta de productos populares con puntuaciones (ratings) altas (Standifird, 2001). En ambos casos, el rol de los canales online es el mismo: ayudar a los consumidores a encontrar los productos que están buscando, sin importar su popularidad. La única diferencia es que, en algunos casos, la cola se ensancha, mientras que, en otros, la cola se alarga (Lee, Lee y Shin, 2011).

Este trabajo proporciona una nueva perspectiva sobre la comprensión del efecto super-hit y el fenómeno de «cola larga» sobre los patrones de consumo de los usuarios online dentro de una comunidad de boca a boca electrónico, estudiando la presencia de los productos a lo largo de todo el espectro de distribución de categorías de productos.

El objetivo y la principal contribución de la investigación es determinar matemáticamente

El objetivo y la principal contribución de la investigación es determinar matemáticamente la presencia de productos super-bit y productos nicho, comparando dos metodologías que miden matemáticamente una distribución de «cola ancha» y una distribución de «cola larga»»

Las metodologías utilizadas son el método de ajuste de la ley de potencia de la distribución del número de productos (factor de la oferta) por el número de comentarios online (factor de la demanda) y el método del codo demarcado por el ajuste de la ley de potencia 
la presencia de productos super-hit y de productos nicho, comparando dos metodologías que miden matemáticamente una distribución de «cola ancha» y una distribución de «cola larga»: el método de ajuste de la ley de potencia de la distribución del número de productos (factor de la oferta) por el número de comentarios online (factor de la demanda), propuesto previamente por Clauset, Shalizi y Newman (2009) y desarrollado por Olmedilla, Martínez-Torres y Toral (2015), y el método del codo demarcado por el ajuste de la ley de potencia.

El resto del artículo está organizado como se describe a continuación. La siguiente sección analiza el trabajo relacionado y el marco conceptual mediante la realización de una revisión de la literatura y el planteamiento de nuestras preguntas de investigación. A continuación, se describe la metodología usada en la investigación, detallando el caso de estudio y la recolección de los datos, así como los dos métodos utilizados para ajustar la distribución de la ley de potencia. Los resultados se presentan posteriormente, para, finalmente, concluir el estudio con la discusión y sus implicaciones.

\section{Enfoque teórico y marco conceptual}

\subsection{Efecto super-hit versus fenómeno de «cola larga»}

Hoy en día, gracias a internet, a las tecnologías digitales y a las redes, un producto se vuelve más apreciado a medida que lo tienen más usuarios, lo que significa que el efecto super-hit está afectando a más productos que antes. No obstante, internet también permite a los consumidores obtener información relacionada con productos menos comunes para un grupo de personas masivo y geográficamente disperso, lo que facilita este efecto de «cola larga» (Olmedilla, Martínez-Torres y Toral, 2015).

En una curva de distribución que sigue una ley de potencia, como la que adquiere la curva de distribución de las ventas o las acciones realizadas por los consumidores en internet, se presenta un grupo pequeño de eventos (productos super-hit) que aparecen frecuentemente en la cabeza, seguidos por un gran conjunto de eventos (productos nicho) que aparecen con menos frecuencia en la cola (Koçaş y Akkan, 2016). Gráficamente esta curva de distribución de la ley de potencia representaría todos los eventos probables clasificados por su frecuencia (véase figura 1). Se observa que en ella los productos super-hit (a la izquierda de la figura 1, en color azul) son los que presentan mayor frecuencia, dando

Hoy en día, gracias a internet, a las tecnologías digitales y a las redes, un producto se vuelve más apreciado a medida que lo tienen más usuarios, lo que significa que el efecto super-bit está afectando a más productos que antes 
lugar a la «cabeza» de la curva, mientras que los productos nicho (a la derecha de la figura 1, en gris) son más numerosos y menos frecuentes, constituyendo la cola de la curva. En este ejemplo, el corte en el eje $x$, marcado en color negro en la figura 1 , se ha seleccionado para que las áreas de la cabeza y de la «cola larga» sean iguales, aunque, según Elberse (2008), el área gris de la curva puede llegar a ser más grande que el área azul a lo largo del tiempo.

Figura 1. Ejemplo de gráfico que representa la distribución de productos/eventos por la ley de potencia

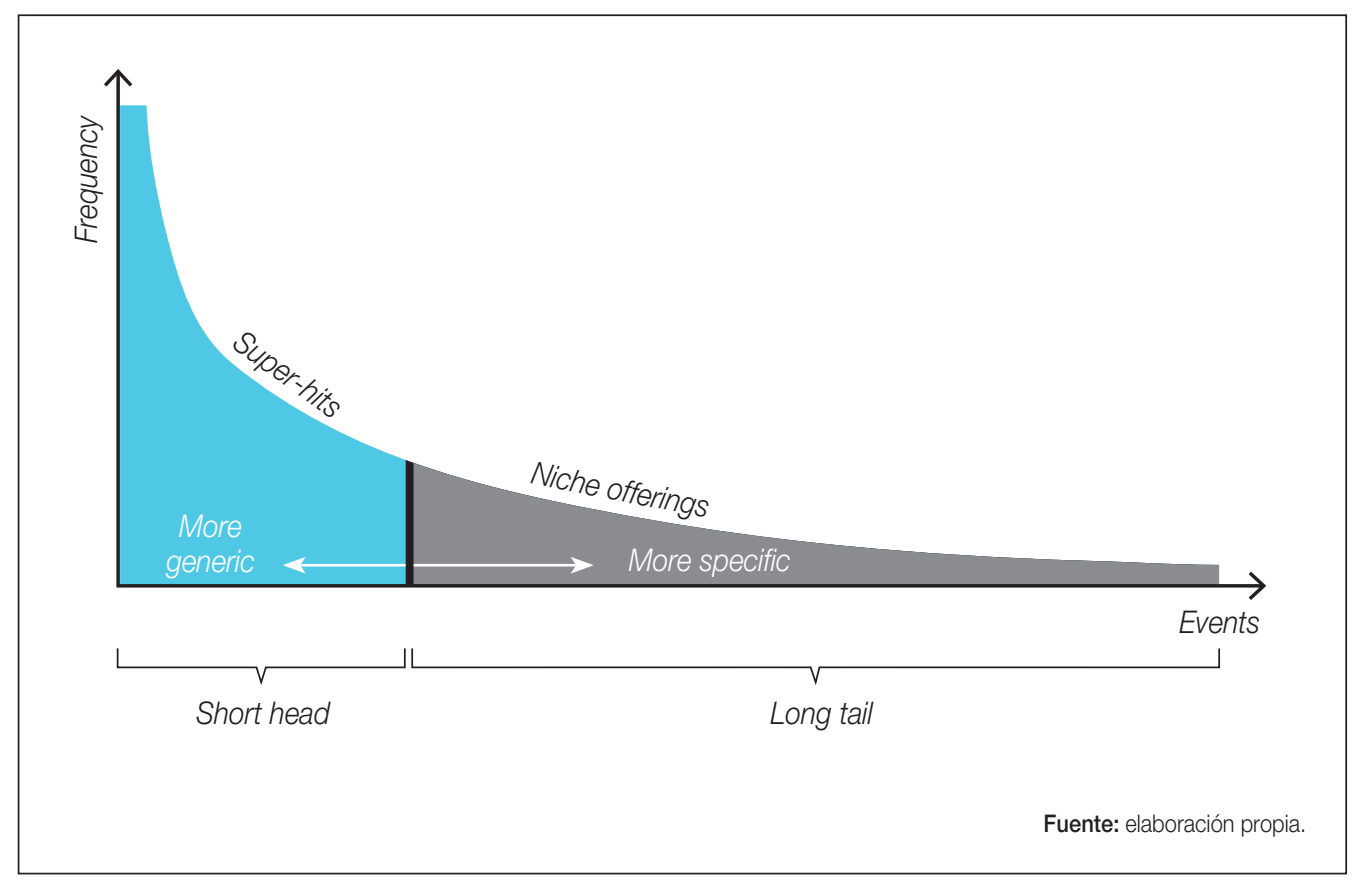

La teoría de la «cola larga» defiende que una gran parte de los consumidores prefieren el contenido popular (super-hit), mientras que solo una minoría busca contenido de nicho (Goel, Broder, Gabrilovich y Pang, 2010). En este sentido, Anderson (2004) teoriza que la cola al final de la curva de distribución es prácticamente diferente a la cabeza y que los consumidores se están moviendo hacia los productos nicho en la cola, ya que esta satisface mejor los intereses que son menos comunes (Anderson, 2008). Además, en algunas áreas, como la música, las películas o los blogs, la cola es teóricamente ilimitada, ya que internet ofrece un espacio de almacenamiento infinito. 


\subsection{Boca a boca electrónico}

Los consumidores comparten cada vez más sus experiencias con un producto o servicio escribiendo comentarios online. La aparición y el impacto del contenido generado por los usuarios dentro de la web ha hecho que el boca a boca clásico avance hacia el boca a boca electrónico (Moe y Trusov, 2011), disminuyendo las barreras de entrada en los mercados de los productos (Martínez-Torres, 2014) y teniendo un fuerte impacto en los juicios sobre los productos al ser la información recibida más accesible. El boca a boca electrónico, o boca-oreja, es ampliamente reconocido como una fuente de información influyente en la toma de decisiones de los consumidores (Park, Lee y Han, 2007). Esta influencia depende de la calidad de la información suministrada, de la calidad de la web que sostiene las opiniones, del nivel de satisfacción del usuario con experiencias previas (Filieri, Alguezaui y McLeay, 2015) y del nivel de confianza que aporte el sitio web (Casado-Aranda, Dimoka y Sánchez-Fernández, 2019).

Estudios existentes sobre el boca a boca electrónico han lidiado principalmente con información sobre productos como películas (Yeap, Ignatius y Ramayah, 2014), libros (Brynjolfsson, Hu y Smith, 2010; Chevalier y Mayzlin, 2006), viajes (Filieri et al., 2015) o música (MoralesArroyo y Pandey, 2010). Muchos han evidenciado su capacidad para encontrar productos nicho, facilitando el fenómeno de «cola larga» al disminuir el costo de la búsqueda de información sobre productos. Se ha evidenciado que este fenómeno depende de las características del producto (Manes y Tchetchik, 2018), de la reputación de la web y de la dirección de la información (Park y Lee, 2009).

\subsection{Ajuste de las distribuciones de la ley de potencia online}

No siempre los procesos medidos alcanzan su punto máximo alrededor de un valor típico; a veces divergen en un amplio rango (Newman, 2005). Así, cuando la varianza y/o la media no son finitas, como ocurre en eventos grandes, el teorema del límite central no predice distribuciones gaussianas, sino distribuciones que se asemejan a las leyes de potencia. Además, a diferencia de las distribuciones normales, las observaciones en las distribuciones de la ley de potencia están muy a la izquierda de la media (de ahí que también se llamen «distribuciones de cola pesada» [Bimpikis y Markakis, 2015]) y algunos valores atípicos representan una cantidad desigual de la producción de la distribución total, poniendo de manifiesto que los eventos en la cola de la distribución tienen más probabilidades de ocurrir (Crawford, Aguinis, Lichtenstein,
A diferencia de las distribuciones normales, las observaciones en las distribuciones de la ley de potencia están muy a la izquierda de la media y algunos valores atípicos representan una cantidad desigual de la producción de la distribución total, poniendo de manifiesto que los eventos en la cola de la distribución tienen más probabilidades de ocurrir 
Davidsson y McKelvey, 2015). Por tanto, las colas extremas son importantes porque dan una idea de con qué frecuencia, en promedio, pueden ocurrir los eventos más grandes. En consecuencia, la distribución de la ley de potencia se considera una herramienta valiosa para medir estas incertidumbres de la información, ya que las distribuciones de Gauss no se pueden usar cuando ocurren a una cierta probabilidad.

Investigaciones anteriores y actuales han examinado si los fenómenos de internet, provocados por la interacción de los usuarios online que intercambian información, están mejor descritos por las distribuciones de la ley de potencia (Barabási y Albert, 1999; Mahanti, Carlsson, Mahanti, Arlitt y Williamson, 2013), demostrando que estas generalmente surgen en los sistemas sociales de internet donde los usuarios expresan sus preferencias entre muchas opciones (por ejemplo, YouTube, comunidades boca a boca electrónicas, Amazon, etc.). Por su parte, Brynjolfsson et al. (2003) argumentan que se puede usar una distribución de la ley de potencia para describir la relación entre el rango de ventas de un producto y la cantidad de ventas.

Numerosos estudios analizan el efecto de los comentarios online sobre las ventas de productos o las elecciones de los consumidores considerando también las características de los comentarios. Una alta proporción de opiniones online implica que hay una elevada participación de los consumidores, por lo que es más probable que un consumidor reciba información sobre un producto, lo que le podría suscitar la intención de comprarlo (Zhang, Zhao, Cheung y Lee, 2014). El volumen de comentarios no significa necesariamente que estos sean positivos. A este respecto, numerosos estudios han argumentado que los comentarios negativos en el boca a boca electrónico tienen un mayor impacto en la intención de compra que los comentarios positivos (Chen, Wang y Xie, 2011), ya que, en general, la información negativa es llamativa e incita a la curiosidad, y los comentarios controvertidos pueden provocar mucha discusión (Shao, 2012). Hay varios artículos relevantes que han verificado un impacto positivo de los comentarios online en las ventas teniendo en cuenta el volumen de comentarios, como los de Maslowska, Malthouse y Viswanathan (2017), Park et al. (2007), Zhou y Duan (2015), Chevalier y Mayzlin (2006) o Zhang, Ma y Cartwright (2013).

En línea con lo anterior, este trabajo se centra en la distribución de la ley de potencia, porque el efecto super-hit y el fenómeno de «cola larga» prevalecen en los patrones de consumo online, donde un número relativamente pequeño de productos muy populares representan la mayoría de las ventas (Brynjolfsson et al., 2010) y la cola de la distribución incluye productos nicho (Anderson, 2008). Para este estudio se utiliza el

Este trabajo se centra en la distribución de la ley de potencia, porque el efecto super-bit y el fenómeno de «cola larga» prevalecen en los patrones de consumo online, donde un número relativamente pequeño de productos muy populares representan la mayoría de las ventas y la cola de la distribución incluye productos nicho. Para este estudio se utiliza el número de comentarios online y no su contenido número de comentarios online y no su contenido. 


\section{Motivación de la investigación y preguntas de investigación}

Investigaciones anteriores han descubierto el impacto de internet en el proceso de descubrimiento de productos por parte de los consumidores y han encontrado evidencias diferentes con respecto al cambio en la distribución de ventas para los distintos productos. Por una parte, Gu et al. (2013) examinan en Amazon el fenómeno de «cola larga» a través del efecto informativo en el contexto del boca a boca electrónico, sugiriendo sus resultados que «el boca a boca electrónico restringe la formación de la "cola larga"» (Gu et al., p. 474). Por otra parte, Chevalier y Mayzlin (2006), utilizando también datos de Amazon, hallan el resultado contrario con relación a las ventas de libros; Zhu y Zhang (2010) defienden que los comentarios online influyen en la difusión y adopción de productos que son menos populares; y Hervas-Draney (2009) muestra que el boca a boca beneficia a los productos más populares y a los consumidores convencionales. Por lo tanto, la primera pregunta de investigación que nos planteamos es:

$\mathbf{P I}_{1}$. ¿Promueve el efecto super-hit o el fenómeno de «cola larga» el boca a boca electrónico en todas las categorías de productos?

Asimismo, se han estudiado diferentes evidencias sobre cómo internet está afectando de manera diferente a la distribución de productos en la actualidad, evaluando la relación entre la «cola larga» y las ventas de productos. Hay investigadores que abogan por el boca a boca electrónico como facilitador del fenómeno de «cola larga» para ayudar a encontrar productos nicho (Dellarocas y Narayan, 2007), mientras otros investigadores describen la distribución de productos como muy desigual, con unos pocos valores extremos acumulados en la cabeza (Zhang et al., 2013). Ambos fenómenos son ampliamente reconocidos en el artículo de Elberse y Oberholzer-Gee (2007), en el que se encuentran pruebas tanto del efecto de «cola larga» como del efecto super-hit en las ventas de vídeos domésticos.

Una distribución atractiva para los datos generados por este tipo de procesos acumulativos es la ley de potencia. Sin embargo, pocos trabajo se han centrado en si la distribución de la ley de potencia es un ajuste plausible a un conjunto de datos de concentración de diferentes surtidos de productos. Por lo tanto, sería conveniente poder modelar la distribución de las categorías de productos con la ley de potencia para discernir si hay eventos de alta frecuencia en la cabeza corta o eventos más frecuentes en la cola. Esto nos lleva a plantear la siguiente pregunta de investigación:

$\mathbf{P I}_{2}$. ¿Es posible que el efecto super-hit coexista con el fenómeno de «cola larga»? 


\section{Metodología de investigación}

\subsection{Caso de estudio y recolección de datos}

La comunidad elegida de boca a boca electrónico para la recopilación y el análisis de datos ha sido Ciao UK, por tratarse de una comunidad de boca a boca electrónico masiva, en la que los usuarios pueden opinar sobre cualquier producto o servicio, y pura, al contrario de Amazon, por ejemplo, que es una página de comercio electrónico que además posee una parte de comunidad boca a boca electrónico. Ciao UK, al igual que otras comunidades del estilo, como TripAdvisor, está formada exclusivamente por una red de usuarios que intercambian ideas en forma de comentarios sobre productos que podría haber anteriormente. Un punto muy importante a la hora de haber elegido Ciao UK es que, al tener 28 categorías de productos de muchos tipos y no estar enfocada en un solo sector, como, por ejemplo, TripAdvisor (turismo) o El Tenedor (restauración), consideramos que los datos extraídos tienen más riqueza y posibilidades de explotación.

Ciao UK es un sitio web con más de 1,3 millones de usuarios que han escrito más de 7 millones de comentarios sobre 1,4 millones de productos (Olmedilla et al. 2015). Tres secciones principales son las que constituyen esta comunidad de boca a boca electrónico: Comentarios, Compras y Mi Ciao, siendo esta última sección la que evalúa la información relevante sobre cada usuario registrado. Hay 28 categorías principales de productos establecidas por Ciao UK, subdivididas, a su vez, en muchas más subcategorías, por lo que los usuarios registrados publican y comparten sus comentarios dentro de una ca-

Ciao UK es un sitio web con más de 1,3 millones de usuarios que han escrito más de 7 millones de comentarios sobre 1,4 millones de productos. Tres secciones principales son las que constituyen esta comunidad de boca a boca electrónico: Comentarios, Compras y Mi Ciao tegoría específica y de una subcategoría relacionada con el tema del comentario. Casi todas las categorías principales tienen cuatro niveles de subcategorías. La figura 2 muestra como ejemplo los cuatro niveles de subcategorías para la categoría principal Viajes. En este caso, la subcategoría nivel 1 agrupa las revisiones por continente; la subcategoría nivel 2, por país; y la subcategoría nivel 3, por ciudad. El nivel 4 ofrece demasiado detalle para la oferta de destino de viaje.

Los usuarios acceden a Ciao UK de forma gratuita, teniendo la opción de registrarse, al crear una cuenta en la que proporcionan información no obligatoria sobre sí mismos (nombre, sexo, edad, país, etc.). Para escribir un comentario de al menos 120 palabras, dentro de la categoría y subcategoría adecuadas, los usuarios deben completar algunos campos, como el título del comentario, el nombre del producto, el cuerpo, que contiene la opinión del usuario, sus ventajas y desventajas, etc. Estos son los datos que se han recopilado 
para este trabajo, formando un subconjunto de casi 45.000 usuarios, 106.000 comentarios aproximadamente de alrededor de 69.000 productos y cerca de 283.000 subcategorías.

Figura 2. Nivel de especificación de la categoría principal Viajes

\begin{tabular}{|c|c|c|c|c|}
\hline Nivel 0 & Nivel 1 & Nivel 2 & Nivel 3 & Nivel 4 \\
\hline Travel & Europe & The Netherlands & Amsterdam & Amsterdam attractions \\
\hline Travel & Europe & The Netherlands & Amsterdam & Amsterdam experience \\
\hline Travel & Europe & The Netherlands & Amsterdam & Amsterdam hotels \\
\hline Travel & Europe & The Netherlands & Amsterdam & Amsterdam pubs, bars and nightlife \\
\hline Travel & Europe & The Netherlands & Amsterdam & Amsterdam restaurants \\
\hline Travel & Europe & The Netherlands & Eindhoven & Eindhoven attractions \\
\hline Travel & Europe & The Netherlands & Maastricht & Maastricht attractions \\
\hline Travel & Europe & The Netherlands & Maastricht & Maastricht experience \\
\hline Travel & Europe & The Netherlands & Other destinations in The Netherlands & The Netherlands experience \\
\hline Travel & Europe & The Netherlands & Other destinations in The Netherlands & The Netherlands hotels \\
\hline Travel & Europe & The Netherlands & Other destinations in The Netherlands & The Netherlands restaurants \\
\hline \multirow[t]{2}{*}{$\begin{array}{l}\text { Categoría } \\
\text { principal }\end{array}$} & & & Subcategorías & \\
\hline & & & & Fuente: elaboración propia. \\
\hline
\end{tabular}

Para la extracción de los datos de la web se ha aplicado el crawler desarrollado en Olmedilla, Martínez-Torres y Toral (2016), usando el lenguaje de programación Python y utilizando una librería de código abierto de extracción web llamada Scrapy. Para comenzar con el ciclo de extracción de todas las categorías, se ha definido un ítem Comentario, que contiene los campos Categoría Principal y Subcategoría que se quieren extraer. Luego, para extraer la información de cada categoría, se ha programado una clase llamada Spider. La clase Spider navega por la página web del producto. Mediante el uso de los selectores XPath para realizar extracciones de datos de la fuente HTML, se ha extraído toda la información sobre las categorías y subcategorías principales. Para ello, se ha llamado al método «response. xpath('text_to_gather').extract)" y se ha creado una lista con todos los elementos (vector) de categorías. A continuación, la información extraída se ha almacenado en una base de datos relacional diseñada en MySQL en los campos Categoría Principal y Subcategoría para la tabla Producto. El lenguaje de consulta SQL se puede utilizar para acceder y rastrear todos los datos almacenados en la base de datos.

\section{Hay 28 categorias principales de productos establecidas por Ciao UK, subdivididas, a su vez, en muchas más subcategorías, por lo que los usuarios registrados publican y comparten sus comentarios dentro de una categoría específica y de una subcategoría relacionada con el tema del comentario}




\subsection{Técnicas cuantitativas}

\subsubsection{Ajuste de la ley de potencia a los datos recopilados}

El primer método, la distribución de la ley de potencia, utilizado por Olmedilla et al. (2015), se ha ampliado para decidir si el conjunto de datos sigue una distribución de la ley de potencia. Según Clauset et al. (2009), muchas de las cosas que los científicos miden tienen un tamaño o «escala» típica; un valor típico en torno al cual se centran las mediciones individuales. Por tanto, como resultado se produce una distribución gaussiana. Sin embargo, no todas las cosas medidas son gaussianas. Los eventos grandes son extremadamente raros dentro de las distribuciones gaussianas. Además, los eventos situados en la cola, que sería la parte de la curva de distribución alejada de la media y que caracterizaría grandes e infrecuentes eventos, tienen más probabilidades de ocurrir en una distribución de la ley de potencia (Clauset et al., 2009). En consecuencia, cuando la probabilidad de alcanzar un valor máximo en torno a un valor típico de alguna cantidad varía en un amplio rango dinámico, dicha cantidad podría ser coherente con una distribución de la ley de potencia (Newman, 2005), que se define como:

$$
P(x)=C x^{-\alpha} \text { para } x>x_{\min }
$$

Donde:

- $P(x)=$ Probabilidad (frecuencia) de que la variable tome el valor $x$.

- $\alpha=$ Exponente de la distribución.

- $x=$ Variable a analizar.

- $C=$ Constante que depende del tipo de evento.

- $x_{\text {min }}=$ Valor mínimo de $x$ sobre el cual comienza el comportamiento de la ley de potencia.

Dado que las leyes de potencia típicamente describen sistemas donde los eventos más grandes son menos que los eventos más pequeños, $\alpha$ se mantiene positivo. Esto confirma que la ley de potencia es una función que decrece monótonamente.

\section{Las leyes de potencia típicamente describen sistemas donde los eventos más grandes son menos que los eventos más pequeños}

Tomando logaritmos en ambos lados de (1), se observa que para una ley de potencia:

$$
\ln (P)=\ln C-\alpha \ln x
$$


en una gráfica con escala logarítmica, la relación entre $\ln (P)$ y $\ln x$ se describe mediante una línea recta cuya pendiente negativa es $\alpha$.

En la práctica, identificar el comportamiento de la ley de potencia es difícil. En muchos casos, es conveniente utilizar la función de distribución acumulada (FDA) de una variable distribuida de la ley de potencia, que se denota como:

$$
P(x)=\int_{x}^{\infty} p(x) d x=\left(\frac{x}{x_{\min }}\right)^{-\alpha+1}
$$

Esencialmente, como resultado de la aplicación de este método de distribución de la ley de potencia, se obtiene el valor de corte $x_{\text {min }}$. Este valor define el punto donde la distribución de la ley de potencia ya no es válida. Por lo tanto, es el punto más allá, donde no hay productos super-hit y, en consecuencia, comienza la cola de la distribución.

Se ha utilizado el lenguaje de programación R para evaluar la presencia de una ley de potencia en los datos mediante el uso de la biblioteca poweRlaw, que sigue el protocolo para ajustar un modelo de la ley de potencia como lo describen Clauset et al. (2009). La función PLFIT se utilizó para estimar el $x_{\min }$ y el $\alpha$ según la bondad de ajuste.

\subsubsection{Encontrar el $x_{\min }$ óptimo según el método del codo}

El segundo método utilizado se basa en el criterio del codo, que es un método visual alternativo para determinar el valor de corte $x_{\text {min }}$, es decir, para determinar qué número de clusters deben elegirse para el clustering de k-medias (Kodinariya y Makwana, 2013).

El método del codo se basa en la relación entre el porcentaje de varianza con respecto al número de clusters para encontrar el número óptimo de estos. En esta relación, representada como un gráfico, se puede observar que las primeras posiciones revelan una pendiente alta debido a que, al tener un número bajo de clusters, es posible obtener un alto porcentaje de varianza entre ellos. Si el número de clusters continúa aumentando en cierto punto, la pendiente disminuye. Esto se debe a que el aumento del número de clusters podría no aumentar la varianza como antes, lo que significa que se ha superado el número óptimo de agrupaciones para la agrupación k-medias, dando un ángulo en la gráfica (Madhulatha, 2012). Extendiendo el criterio del codo al caso de una distribución de la ley de potencia, el problema consiste en encontrar el punto $x_{\min }$, donde el «codo" puntiagudo es claramente visible en la gráfica (véase figura 3). 
Figura 3. Aplicación del criterio de codo a la distribución de potencia

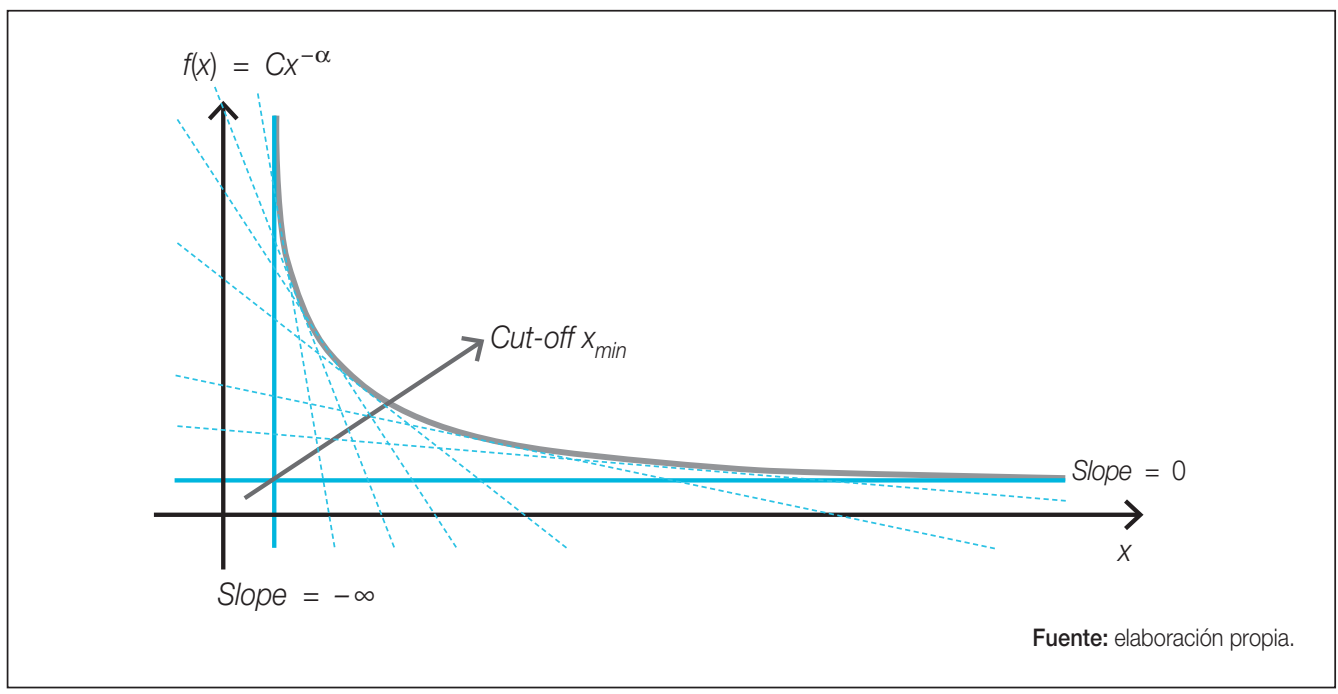

La figura 3 ilustra la selección del criterio del codo basándose en la elección del valor con una disminución brusca en la pendiente de la tangente. Dada la función $f(x)=C x^{-\alpha}$, la línea de la tangente en el punto $\left(x_{0}, f\left(x_{0}\right)\right)$ es la línea recta única que pasa por ese punto y tiene la misma pendiente que la gráfica en ese punto, que se define por el valor derivado $f^{\prime}\left(x_{0}\right)$ :

$$
f^{\prime}\left(x_{0}\right)=\lim _{x \rightarrow x_{0}} \frac{f(x)-f\left(x_{0}\right)}{x-x_{0}}
$$

Las líneas continuas azules de la figura 3 representan la tangente inicial y final, correspondientes a los valores de pendiente de $-\infty$ y 0 . Las líneas punteadas azules intermedias representan todos los valores intermedios posibles de la pendiente. $x_{\min }$ es el punto de la pendiente promedio entre las dos asíntotas del gráfico, dado por el valor - 1, que es el punto desde el cual la curva pasa de disminuir muy rápido a disminuir muy lento.

\section{Resultados}

Siguiendo la metodología propuesta, se ha ajustado una distribución de la ley de potencia a la curva de la distribución de subcategorías creadas por los usuarios que han publicado comentarios en las 28 categorías principales distinguidas por Ciao UK. El resultado recopilado se muestra en la figura 4 , que ilustra dicha distribución de los comentarios en las categorías principales. 
Figura 4. Distribución de comentarios publicados en las 28 categorías principales

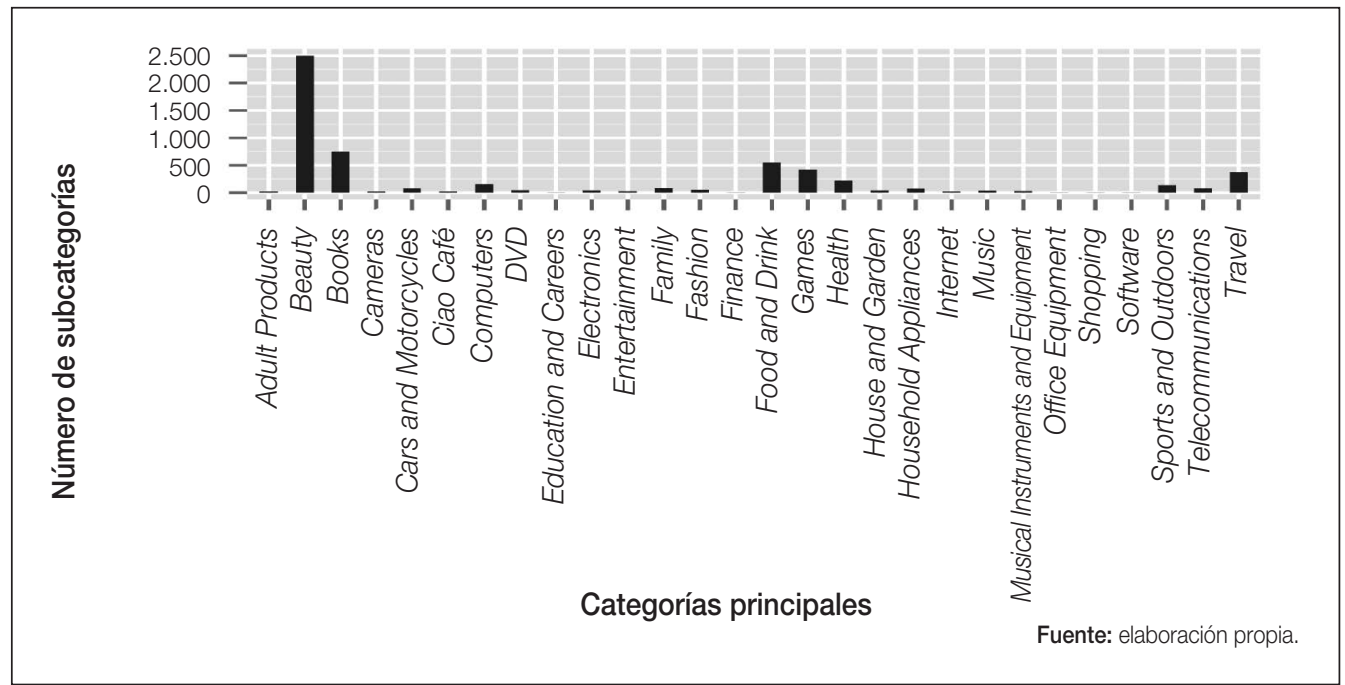

Todas las distribuciones obtenidas muestran que hay algunas subcategorías muy populares, mientras que otras solo están respaldadas por una pequeña cantidad de comentarios. Al aplicar los dos métodos descritos en la sección anterior, nuestro objetivo es determinar la presencia del efecto super-hit y la longitud de la cola para cada una de las 28 categorías principales de Ciao UK.

El cuadro 1 describe en detalle los parámetros de «cola larga» de las 28 categorías principales de acuerdo con el ajuste de la ley de potencia. La segunda y la tercera columnas muestran los valores $x_{\min }$ y $\alpha$ del ajuste de la distribución de la ley de potencia, respectivamente. La cuarta columna corresponde a la bondad del ajuste, el p-valor. La quinta columna muestra la longitud de la cola, calculada como el número de subcategorías que tienen un número de comentarios por debajo del umbral $x_{\min }$, es decir, el número de subcategorías que no forman parte de la distribución de la ley de potencia ajustada. Las columnas sexta y séptima representan las áreas de la cabeza y la cola de la distribución, respectivamente, calculadas también usando el valor $x_{\text {min }}$.

Los valores de $x_{\min }$ distinguen entre aquellas subcategorías que forman parte de la cabeza de la distribución de la ley de potencia (productos super-hit, por encima de $x_{\min }$ ) y aquellos que pertenecen a la cola (productos nicho, por debajo de $x_{\min }$ ). El exponente $\alpha$ determina la forma de la distribución de la ley de potencia. El ajuste tiene un p-valor asociado, de modo que en aquellos casos en que el p-valor calculado sea considerablemente inferior a 0,05, se rechaza la hipótesis nula, lo que significa que dicha categoría no sigue una distribución de la ley de potencia y, por lo tanto, no existe ningún efecto super-hit. Pero si el p-valor 
resultante es mayor que 0,05 , la hipótesis nula no puede ser rechazada y, por lo tanto, la dis-

El cuadro 1 describe en detalle los parámetros de «cola larga» de las 28 categorías principales de acuerdo con el ajuste de la ley de potencia de la ley de potencia, mostrando entonces un efecto de super-hit. Finalmente, la columna de porcentaje del área principal indica el área contenida a la izquierda del punto de corte de $x_{\min }$, que incluye los productos dominantes o los más vendidos. La columna de porcentaje del área de la cola incorpora la cola, es decir, la región a la derecha del punto de corte de $x_{\text {min }}$.

Cuadro 1. Parámetros de "cola larga» de las 28 categorías principales de Ciao UK según el método de la ley de potencia

\begin{tabular}{l|c|c|c|c|c|c|}
\hline Principales categorías & $x_{\min }$ & $\alpha$ & p-valor & $\begin{array}{c}\text { Longitud de } \\
\text { la cola (\%) }\end{array}$ & $\begin{array}{c}\text { Área de la } \\
\text { cabeza (\%) }\end{array}$ & $\begin{array}{c}\text { Área de la } \\
\text { cola (\%) }\end{array}$ \\
\hline Adult Products & 3 & 2,97 & 0,602 & 68,75 & 46,03 & 53,97 \\
\hline Beauty & 3 & 2,02 & 0,000 & 65,91 & 74,32 & 25,68 \\
\hline Books & 7 & 2,20 & 0,011 & 76,03 & 72,02 & 27,98 \\
\hline Cameras & 4 & 1,63 & 0,231 & 10,53 & 98,04 & 1,96 \\
\hline Cars and Motorcycles & 3 & 1,84 & 0,047 & 30,00 & 89,88 & 10,12 \\
\hline Ciao Café & 18 & 1,62 & 0,085 & 20,00 & 98,27 & 1,73 \\
\hline Computers & 5 & 1,74 & 0,002 & 55,48 & 91,29 & 8,71 \\
\hline DVD & 5 & 1,78 & 0,435 & 52,17 & 91,13 & 8,87 \\
\hline Education and Careers & 8 & 1,53 & 0,098 & 0,00 & 100 & 0 \\
\hline Electronics & 20 & 2,21 & 0,683 & 55,56 & 88,74 & 11,26 \\
\hline Entertainment & 18 & 2,72 & 0,940 & 56,52 & 80,92 & 19,08 \\
\hline Fasmily & 57 & 3,50 & 0,520 & 84,88 & 56,41 & 43,59 \\
\hline
\end{tabular}




\begin{tabular}{|c|c|c|c|c|c|c|}
\hline Principales categorías & $x_{\min }$ & $\alpha$ & p-valor & $\begin{array}{l}\text { Longitud de } \\
\text { la cola ( } \%)\end{array}$ & $\begin{array}{l}\text { Área de la } \\
\text { cabeza (\%) }\end{array}$ & $\begin{array}{c}\text { Área de la } \\
\text { cola (\%) }\end{array}$ \\
\hline \multicolumn{7}{|l|}{$\triangleright$} \\
\hline Finance & 118 & 3,24 & 0,590 & 50,00 & 82,78 & 17,22 \\
\hline Food and Drink & 2 & 1,81 & 0,086 & 47,55 & 87,76 & 12,24 \\
\hline Games & 9 & 2,13 & 0,171 & 80,52 & 73,59 & 26,41 \\
\hline Health & 17 & 2,39 & 0,064 & 86,70 & 67,32 & 32,68 \\
\hline House and Garden & 19 & 2,01 & 0,683 & 35,90 & 94,82 & 5,18 \\
\hline Household Appliances & 71 & 3,50 & 0,703 & 81,33 & 52,62 & 47,38 \\
\hline Internet & 13 & 1,50 & 0,048 & 11,76 & 99,45 & 0,55 \\
\hline Music & 8 & 3,50 & 0,179 & 71,05 & 60,18 & 39,82 \\
\hline $\begin{array}{l}\text { Musical Instruments and } \\
\text { Equipment }\end{array}$ & 8 & 2,70 & 0,711 & 83,33 & 45,28 & 54,72 \\
\hline Office Equipment & 4 & 1,52 & 0,161 & 0,00 & 100 & 0 \\
\hline Shopping & 11 & 1,50 & 0,458 & 0,00 & 100 & 0 \\
\hline Software & 7 & 1,52 & 0,538 & 20,00 & 98,13 & 1,87 \\
\hline Sports and Outdoors & 5 & 2,21 & 0,036 & 68,46 & 72,56 & 27,44 \\
\hline Telecommunications & 71 & 2,22 & 0,020 & 90,00 & 84,10 & 15,90 \\
\hline Travel & 3 & 1,98 & 0,157 & 53,23 & 83,12 & 16,88 \\
\hline
\end{tabular}

El cuadro 2 describe los resultados del mismo análisis para las 28 categorías principales, pero utilizando el método del codo. La primera columna muestra los valores de $x_{\min }$, que corresponden al punto de inflexión, es decir, el punto desde el cual la función pasa de disminuir muy rápido a disminuir muy lentamente. En consecuencia, la función se recorre de derecha a izquierda, de $x_{\max } a-1$, hasta el primer punto/valor que cumple la condición de búsqueda. Este es el punto de la pendiente promedio entre las dos asíntotas de la gráfica. La tercera 
columna muestra la longitud de la cola, que se halló utilizando el punto de inflexión calculado. Finalmente, la cuarta y quinta columnas representan las áreas de la cabeza y la cola de la distribución, respectivamente, calculadas utilizando el punto de inflexión obtenido.
El cuadro 2 describe los resultados del mismo análisis para las 28 categorías principales, pero utilizando el método del codo

Cuadro 2. Parámetros de "cola larga» de las 28 categorías principales de Ciao UK según el método del codo

\begin{tabular}{|c|c|c|c|c|}
\hline Principales categorías & $x_{\min }$ & $\begin{array}{l}\text { Longitud de } \\
\text { la cola (\%) }\end{array}$ & $\begin{array}{l}\text { Área de la } \\
\text { cabeza (\%) }\end{array}$ & $\begin{array}{c}\text { Área de la } \\
\text { cola (\%) }\end{array}$ \\
\hline Adult Products & 2 & 56,25 & 63,49 & 36,51 \\
\hline Beauty & 2 & 50,50 & 80,60 & 19,40 \\
\hline Books & 5 & 69,51 & 77,23 & 22,77 \\
\hline Cameras & 4 & 10,53 & 98,04 & 1,96 \\
\hline Cars and Motorcycles & 2 & 18,75 & 95,63 & 4,37 \\
\hline Ciao Café & 8 & 13,33 & 99,19 & 0,81 \\
\hline Computers & 4 & 50,32 & 92,52 & 7,48 \\
\hline DVD & 4 & 50,00 & 93,51 & 6,49 \\
\hline Education and Careers & 8 & 0,00 & 100,00 & 0,00 \\
\hline Electronics & 24 & 62,22 & 81,47 & 18,53 \\
\hline Entertainment & 23 & 70,83 & 62,31 & 37,69 \\
\hline Family & 65 & 87,21 & 46,34 & 53,66 \\
\hline Fashion & 2 & 35,71 & 77,92 & 22,08 \\
\hline Finance & 149 & 66,67 & 54,04 & 45,96 \\
\hline Food and Drink & 2 & 210,31 & 87,76 & 12,24 \\
\hline
\end{tabular}




\begin{tabular}{|c|c|c|c|c|}
\hline Principales categorias & $x_{\min }$ & $\begin{array}{l}\text { Longitud de } \\
\text { la cola (\%) }\end{array}$ & $\begin{array}{l}\text { Área de la } \\
\text { cabeza (\%) }\end{array}$ & $\begin{array}{c}\text { Área de la } \\
\text { cola (\%) }\end{array}$ \\
\hline \multicolumn{5}{|l|}{$\triangleright$} \\
\hline Games & 11 & 83,61 & 69,11 & 30,89 \\
\hline Health & 23 & 90,37 & 59,76 & 40,24 \\
\hline House and Garden & 10 & 30,77 & 95,64 & 4,36 \\
\hline Household Appliances & 82 & 85,33 & 43,82 & 56,18 \\
\hline Internet & 10 & 5,88 & 99,76 & 0,24 \\
\hline Music & 5 & 68,42 & 68,86 & 31,14 \\
\hline Musical Instruments and Equipment & 9 & 86,67 & 38,58 & 61,42 \\
\hline Office Equipment & 8 & 16,67 & 97,11 & 2,89 \\
\hline Shopping & 107 & 33,33 & 85,56 & 14,44 \\
\hline Software & 7 & 20,00 & 98,13 & 1,87 \\
\hline Sports and Outdoors & 7 & 77,69 & 63,88 & 36,12 \\
\hline Telecommunications & 83 & 91,25 & 79,21 & 20,79 \\
\hline Travel & 2 & 36,83 & 88,47 & 11,53 \\
\hline
\end{tabular}

Fuente: elaboración propia

Ambos métodos se han comparado en el cuadro 3 para probar su coincidencia o no, identificando la presencia de la «cola larga» y considerando los criterios de área y longitud. El resultado de esta comparación se muestra en la última columna del cuadro 3 . Un valor verdadero significa que ambos métodos están de acuerdo con la presencia o ausencia de la «cola larga», un valor falso significa que ambos métodos están en desacuerdo y un valor incierto
Un valor verdadero significa que ambos métodos están de acuerdo con la presencia o ausencia de la «cola larga», un valor falso significa que ambos métodos están en desacuerdo y un valor incierto significa que ambos métodos generan resultados contradictorios 
significa que ambos métodos generan resultados contradictorios. La toma de decisiones se ha llevado a cabo aplicando al área debajo de la cola o longitud de la cola la relación de unión 80:20. Es decir, siempre que el área o la longitud de la cola estén por debajo del $20 \%$ del valor máximo, se considera que no hay «cola larga». De lo contrario, se acepta la presencia de la «cola larga».

Cuadro 3. Validación de la presencia de "cola larga» a través de la consistencia de reglas de decisión

\begin{tabular}{|c|c|c|c|c|c|}
\hline \multirow{2}{*}{ Principales categorías } & \multicolumn{2}{|c|}{ Áreas } & \multicolumn{2}{|c|}{ Longitud de la cola } & \multirow{2}{*}{$\begin{array}{c}\text { Comparación } \\
\text { Áreas } \\
\text { versus cola }\end{array}$} \\
\hline & $\begin{array}{l}\text { Ley de } \\
\text { potencia }\end{array}$ & Codo & $\begin{array}{l}\text { Ley de } \\
\text { potencia }\end{array}$ & Codo & \\
\hline Adult Products & Fuerte/Sí & Sí & Sí & Sí & Verdadero \\
\hline Beauty & Sí & Sí & Sí & Sí & Verdadero \\
\hline Books & Sí & Sí & Fuerte/Sí & Sí & Verdadero \\
\hline Cameras & No & No & No & No & Verdadero \\
\hline Cars and Motorcycles & No & No & Sí & Sí & Falso \\
\hline Ciao Café & No & No & Sí & No & Incierto \\
\hline Computers & No & No & Sí & Sí & Falso \\
\hline DVD & No & No & Sí & Sí & Falso \\
\hline Education and Careers & No & No & No & No & Verdadero \\
\hline Electronics & No & Sí & Sí & Sí & Incierto \\
\hline Entertainment & Sí & Sí & Sí & Sí & Verdadero \\
\hline Family & Sí & Fuerte/Sí & Fuerte/Sí & Fuerte/Sí & Verdadero \\
\hline Fashion & Sí & Sí & Sí & Sí & Verdadero \\
\hline Finance & Sí & Sí & Sí & Sí & Verdadero \\
\hline
\end{tabular}




\begin{tabular}{|c|c|c|c|c|c|}
\hline \multirow{2}{*}{ Principales categorías } & \multicolumn{2}{|c|}{ Áreas } & \multicolumn{2}{|c|}{ Longitud de la cola } & \multirow{2}{*}{$\begin{array}{c}\text { Comparación } \\
\text { Áreas } \\
\text { versus cola }\end{array}$} \\
\hline & $\begin{array}{l}\text { Ley de } \\
\text { potencia }\end{array}$ & Codo & $\begin{array}{l}\text { Ley de } \\
\text { potencia }\end{array}$ & Codo & \\
\hline \multicolumn{6}{|l|}{$\triangleright$} \\
\hline Food and Drink & Sí & Sí & Sí & Sí & Verdadero \\
\hline Games & Sí & Sí & Fuerte/Sí & Fuerte/Sí & Verdadero \\
\hline Health & Sí & Sí & Fuerte/Sí & Fuerte/Sí & Verdadero \\
\hline House and Garden & No & No & Sí & Sí & Falso \\
\hline Household Appliances & Sí & Fuerte/Sí & Fuerte/Sí & Fuerte/Sí & Verdadero \\
\hline Internet & No & No & No & No & Falso \\
\hline Music & Sí & Sí & Sí & Sí & Verdadero \\
\hline $\begin{array}{l}\text { Musical Instruments } \\
\text { and Equipment }\end{array}$ & Fuerte/Sí & Fuerte/Sí & Fuerte/Sí & Fuerte/Sí & Verdadero \\
\hline Office Equipment & No & No & No & No & Verdadero \\
\hline Shopping & No & Sí & No & Sí & Incierto \\
\hline Software & No & No & Sí & Sí & Falso \\
\hline Sports and Outdoors & Sí & Sí & Sí & Fuerte/Sí & Verdadero \\
\hline Telecommunications & Sí & Sí & Sí & Fuerte/Sí & Verdadero \\
\hline Travel & Sí & No & Sí & Sí & Incierto \\
\hline
\end{tabular}

Las figuras 5.1 y 5.2 representan algunas de las distribuciones para las categorías principales mostradas en el cuadro 3. Las gráficas muestran una distribución de la ley de potencia ajustada a la curva de la distribución de subcategorías de productos. En consecuencia, el eje $y$ corresponde al volumen de comentarios (número de comentarios escritos por los usuarios) y el eje $x$ representa todas las subcategorías de productos de cada una de las 28 categorías principales de productos distinguidas por Ciao UK. 
En ambas figuras, el valor de $x_{\min }$ está demarcado por una línea gris horizontal que representa su valor calculado por el método del codo y por una línea azul horizontal que representa su valor calculado por el método de la ley de potencia. De acuerdo con el cuadro 3, observamos que 15 de las 28 categorías principales (Adult Products [Productos para Adultos], Beauty [Belleza], Books [Libros], Entertainment [Entretenimiento], Family [Familia], Fashion [Moda], Finance [Finanzas], Food and Drinks [Alimentos y Bebidas], Games [Juegos], Health [Salud], Household Appliances [Electrodomésticos], Music [Música], Musical Instruments and Equipment [Instrumentos Musicales y Equipos], Sports and Outdoors [Deportes y Aire Libre] y Telecommunications [Telecomunicaciones]) exhiben claramente un comportamiento de "cola larga», ya que ambos criterios se cumplen utilizando los dos métodos propuestos.

Figura 5.1. Distribución de los comentarios para algunas de las principales categorías en Ciao UK
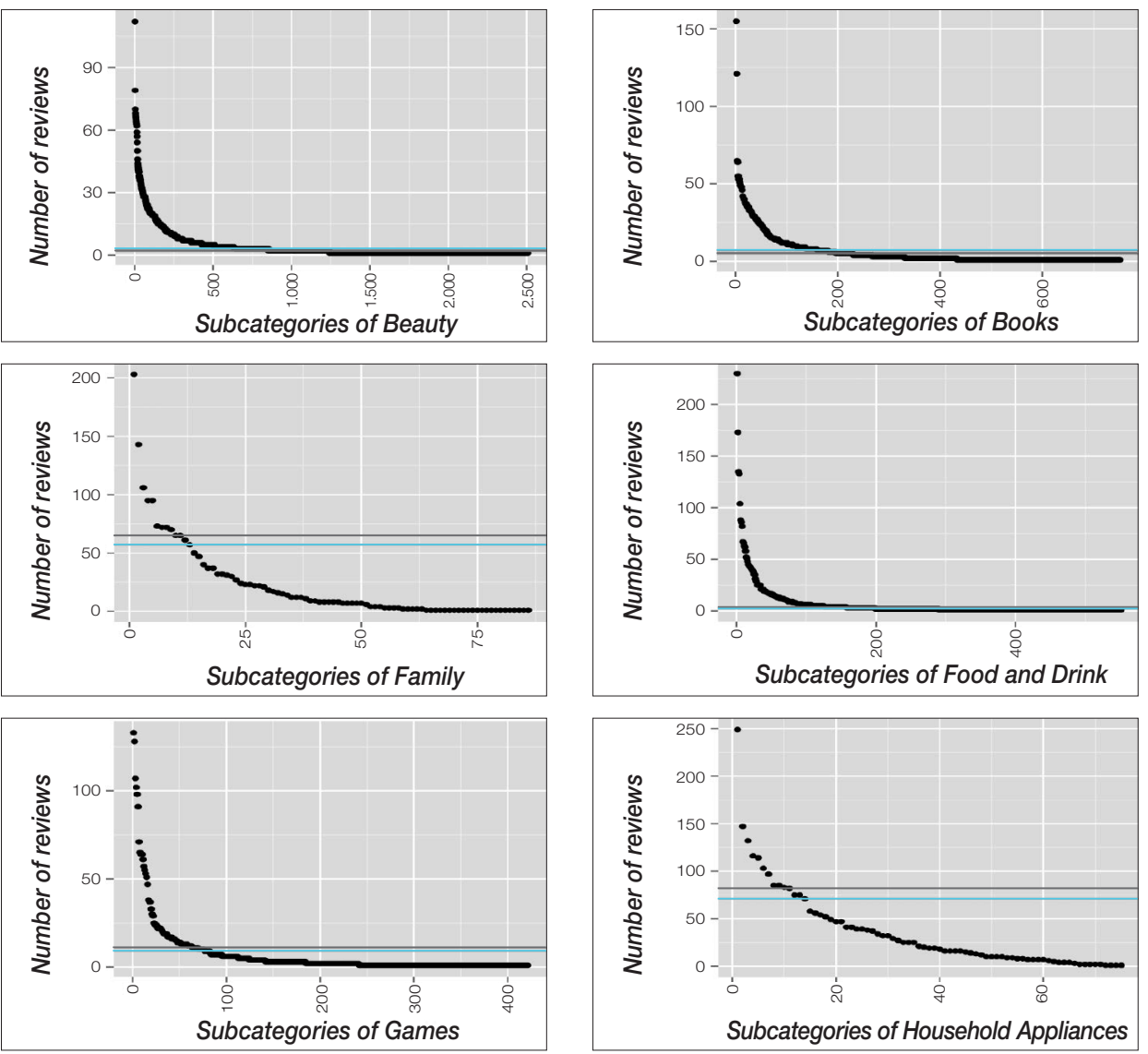

Fuente: elaboración propia. 
Figura 5.2. Distribución de los comentarios para algunas de las principales categorías en Ciao UK

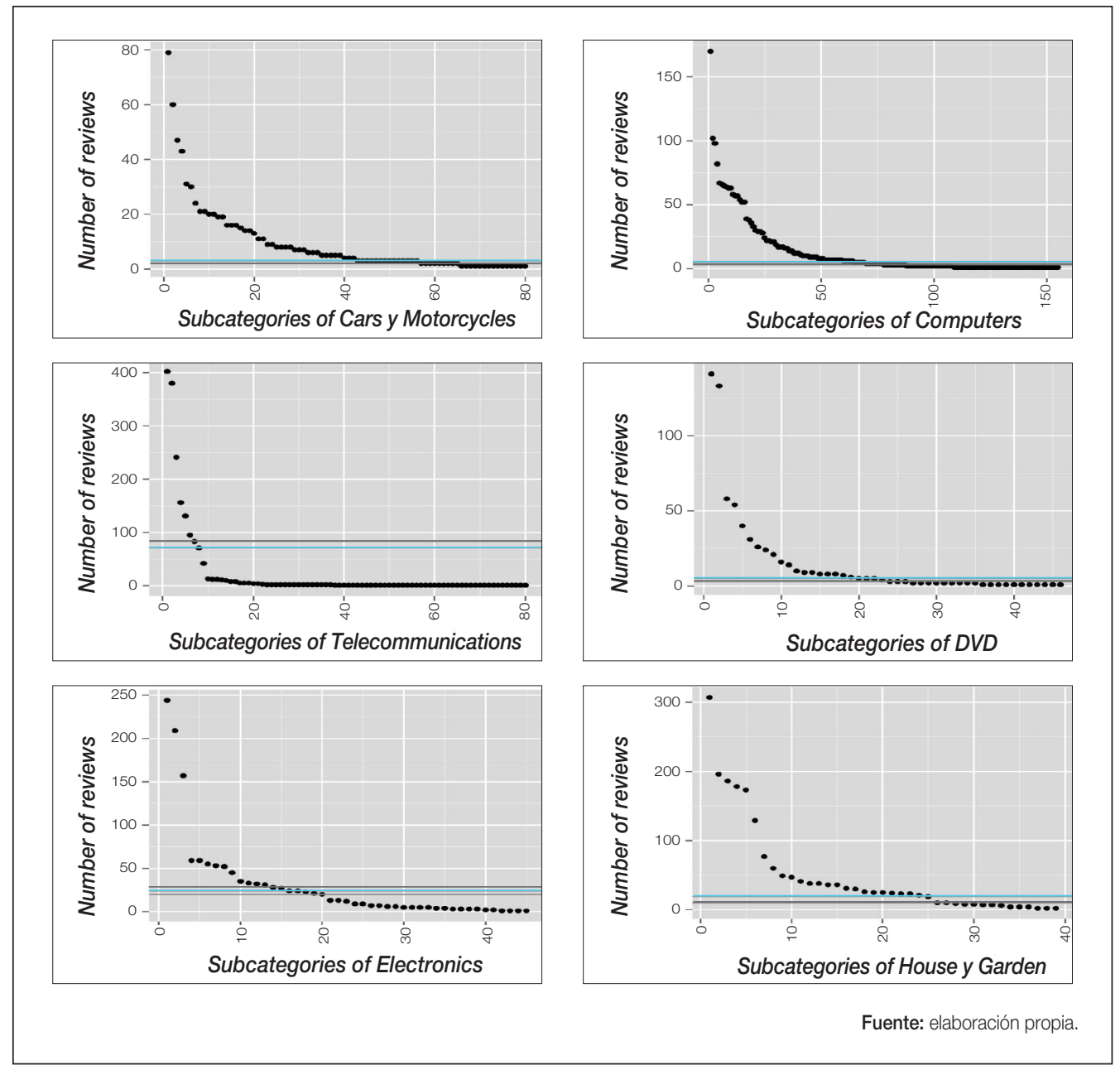

Con respecto al efecto super-hit, los p-valor del cuadro 1 revelan que 7 de las 28 categorías del conjunto de datos (Beauty [Belleza], Books [Libros], Cars and Motorcycles [Automóviles y Motocicletas], Computers [Computadoras], Internet [Internet], Sports and Outdoor [Deportes y Aire Libre] y Telecommunications [Telecomunicaciones]) no obedecen a una distribución de la ley de potencia (véase figura 5.2). Sus p-valor son lo suficientemente pequeños para que el modelo de la ley de potencia pueda ser descartado firmemente, según su bondad de ajuste. Por otra parte, y de acuerdo con sus p-valor, las 21 categorías restantes pueden ser consistentes con una distribución de la ley de potencia. En consecuencia, y respondiendo a $\mathrm{Pl}_{1}$, el boca a boca electrónico promueve tanto el efecto de super-hit como el fenómeno de "cola larga», aunque el efecto super-hit es más frecuente en las 28 categorías consideradas. 
Entre los 21 casos con el efecto super-hit, hay 6 casos sin «cola larga» (DVD, Education and Careers [Educación y Carreras], Cameras [Cámaras], House and Garden [Casa y Jardín], Office Equipment [Material de Oficina] y Software), 11 con "cola larga» (Adult Products [Productos para Adultos], Entertainment [Entretenimiento], Family [Familia], Finance [Finanzas], Food and Drinks [Alimentos y Bebidas], Games [Juegos], Health [Salud], Household Appliances [Electrodomésticos], Music [Música], Musical Instruments and Equipment [Instrumentos y Equipos Musicales] y Fashion [Moda]) y, finalmente, 4 de ellos que no está claro si exhiben una «cola larga» o no (Ciao Café [Ciao Café], Shopping [Compras], Travel [Viajes] y Electronics [Electrónica]). Estos hallazgos responden a la $\mathrm{Pl}_{2}$, al mostrar que es posible que el efecto de super-hit coexista con el fenómeno de la «cola larga» dependiendo de las categorías de productos.

\section{Discusión e implicaciones}

\subsection{Discusión}

Este artículo contribuye a la creciente investigación sobre cómo internet influye en el efecto super-hit y la formación de «cola larga» a través de sus datos, y, más específicamente, del contenido generado por el usuario. Los resultados sugieren, como establecen Park y Lee (2009), que es importante considerar el impacto de las comunidades de boca a boca electrónico en el proceso de descubrimiento de productos por parte de los consumidores, ya que muchos productos solo se venden online y sus consumidores son más propensos a utilizar los comentarios online como fuente principal de información. La interacción de los usuarios que intercambian información sobre los productos dentro de las comunidades de boca a boca electrónico crea una dinámica compleja en la demanda.

La literatura existente relacionada con la «cola larga» ha demostrado que el boca a boca electrónico tiene un impacto positivo en las ventas (Elberse y Oberholzer-Gee, 2007; HervasDraney, 2009). Además, otros estudios de boca a boca electrónico han encontrado evidencia del efecto super-hit, apoyando que este boca a boca promueve las ventas de productos populares, por lo que la parte principal de la distribución de ventas se vuelve más gruesa (Standifird, 2001). La premisa de nuestro estudio es aclarar estas dos escuelas de pensamiento. En este sentido, se ha encontrado evidencia de que el boca a boca electrónico es un promotor tanto de uno como de otro dependiendo de las categorías de productos. Sin embargo, y siguiendo estudios previos (Standifird, 2001), la presencia del efecto super-hit está más extendida que la "cola larga», lo que indica que el fenómeno de "cola larga» está estrechamente relacionado con ciertas categorías de productos donde las revisiones difunden nuevos conocimientos sobre artículos o subcategorías desconocidas para la mayoría de los usuarios. Además, también encontramos evidencia de que el fenómeno de «cola larga» está estrechamente relacionado con ciertas categorías de productos. Los hallazgos están respaldados por dos métodos cuantitativos (Clauset et al., 2009; Kodinariya y Makwana, 2013) que proporcionan evidencia de forma matemática sobre el efecto super-hit y el fenómeno de «cola larga». 
En línea con los estudios de Dellarocas y Narayan (2007) y Elberse y Oberholzer-Gee (2007), demostramos que el fenómeno de «cola larga» es capaz de coexistir con el efecto super-hit dependiendo de la categoría de producto. No es sorprendente que internet haga que a los consumidores les resulte más fácil recopilar información a bajo costo y les permita confiar más en la experiencia de otros usuarios cuando evalúan productos (Filieri et al., 2015). Una vez más, la gran disponibilidad de comentarios online sobre muchos tipos de productos dentro de las comunidades de boca a boca electrónico es el factor explicativo de este fenómeno, dado que este canal online permite a los clientes encontrar más productos nicho que se ajusten a sus preferencias o promover la popularidad de los productos, llevando a una situación de «el rico se enriquece más», favoreciendo los productos que ya son muy populares en ciertas categorías de productos y también a una distribución más igualitaria que facilita la formación de la «cola larga» entre otras categorías de productos. La coexistencia de productos super-hit y productos nicho también significa que el boca a boca electrónico probablemente ha expandido una cola corta original. Casos como la música, el entretenimiento o la moda son ejemplos de categorías de productos donde los mercados online ofrecen la difusión de nuevas opciones desconocidas de otra forma para los clientes. Además, los comentarios online proporcionan no solo la descripción de nuevas opciones, sino también la calidad de la experiencia. La mayoría de los comentarios online se centran más en los sentimientos, en las percepciones y en la satisfacción que en las características objetivas de los productos. Por lo tanto, los canales online ofrecen información más completa que la que se puede encontrar en catálogos o publicidad.

\subsection{Implicaciones para la gestión}

Hoy en día, cualquier marca o empresa puede llegar sin costo alguno a muchos consumidores potenciales a través de internet. Esto hace que sea rentable invertir tiempo y esfuerzo para crear productos que puedan interesar incluso a un pequeño segmento de ellos. Por lo tanto, una estrategia para aumentar la variedad e información de productos nicho podría permitir a los consumidores descubrir aquellos que, de otra manera, no estarían disponibles.

Por ello, un minorista debe comprender el impacto del boca a boca electrónico en la distribución de diferentes productos para ofrecer la variedad precisa y administrar el inventario. En este sentido, nuestros resultados muestran qué productos de la distribución de categorías

\section{Una estrategia para aumentar la variedad e información de productos nicho podría permitir a los consumidores descubrir aquellos que, de otra manera, no estarian disponibles}

Un minorista debe comprender el impacto del boca a boca electrónico en la distribución de diferentes productos para ofrecer la variedad precisa y administrar el inventario 
de productos forman la «cola larga» o son super-hits, lo que permite especificar los casos en detalle. Por tanto, es posible que los minoristas online deban prestar más atención si su objetivo es capturar el mercado masivo, adquiriendo y administrando en este caso clientes mediante el uso de los productos más populares o super-hit y centrándose también en la comercialización de esos productos de gran éxito. Por el contrario, si su objetivo es utilizar estrategias de «cola larga» para vender una gama más amplia de productos en cantidades más pequeñas, deberían centrarse en ampliar su surtido con más productos nicho.

Asimismo, las empresas pueden aprovechar los resultados para desarrollar mejores estrategias de marketing en publicidad y en promoción de productos que los consumidores tienen más probabilidades de comprar en el futuro.
Las empresas pueden aprovechar los resultados para desarrollar mejores estrategias de marketing en publicidad y promoción de productos que los consumidores tendrán más probabilidades de comprar en el futuro

\subsection{Limitaciones de la investigación}

La principal limitación podría ser que la metodología se ha implementado en una sola comunidad de boca a boca electrónico. Sin embargo, sería posible extender los dos métodos (ajuste de la ley de potencia y método del codo) para ajustar la distribución de productos en otras comunidades de boca a boca electrónico. Otra limitación metodológica podría ser la muestra del conjunto de datos, debido a que Ciao UK no representa a toda la población, sino a un subconjunto particular de usuarios online. Además, los usuarios de Ciao UK pueden tener varios perfiles, o incluso los perfiles de los usuarios pueden ser empleados por varias personas. Si bien esta limitación no influye en los resultados, debe entenderse que se aplican a un determinado conjunto de usuarios online. Una limitación adicional es el hecho de que un usuario no tiene que proporcionar ninguna prueba de compra del producto para que se le permita escribir un comentario. Sin embargo, al escribir comentarios, los usuarios consiguen beneficios de estatus y económicos, y, para obtenerlos, pueden no estar interesados en ser deshonestos. Finalmente, otra limitación es la ausencia de datos de ventas, ya que este documento se basa en el volumen de comentarios online, que es la mejor información disponible dentro de una comunidad de boca a boca electrónico. A pesar de ello, este estudio se puede contextualizar en que analiza la diversidad de los productos que supuestamente han sido comprados antes de que el usuario escriba un comentario online.

\subsection{Trabajo futuro}

Investigaciones futuras pueden extender los hallazgos caracterizando cuáles son los productos nicho de la «cola larga» y cuáles los productos super-hit. El objetivo sería descubrir 
algunos patrones comunes entre los productos nicho mediante la creación de modelos de redes sociales o reglas de asociación y descubrir grupos de productos nicho que pueden representar un objetivo rentable para los minoristas. Además, los productos pueden clasificarse como productos de búsqueda y experiencia, por lo que, como trabajo futuro, se podría analizar qué tipo de producto es más propenso a exhibir los dos comportamientos estudiados. También sería interesante poder probar la validez de los modelos presentados en otras plataformas de boca a boca electrónico con información específica disponible de ventas (por ejemplo, Amazon).

\section{Referencias bibliográficas}

Anderson, C. (2004). The long tail. Wired Magazine, 12 (10), 170-177.

Anderson, C. (2008). Long Tail: Why the Future of Business is Selling Less of More. New York: Hyperion Books.

Barabási, A. L. y Albert, R. (1999). Emergence of scaling in random networks. Science, 286, 509-512.

Bimpikis, K. y Markakis, M. G. (2015). Inventory pooling under heavy-tailed demand. Management Science, 62(6), 1.800-1.813.

Brynjolfsson, E., Hu, Y. y Smith, M. (2003). Consumer surplus in the digital economy: estimating the value of increased product variety at online booksellers. Management Science, 49(11), 1.580-1.596.

Brynjolfsson, E., Hu, Y. y Smith, M. (2010). Research commentary-long tails vs. superstars: the effect of information technology on product variety and sales concentration patterns. Information Systems Research, 21(4), 736-747.

Casado-Aranda, L. A., Dimoka, A. y SánchezFernández, J. (2019). Consumer proces- sing of online trust signals: a neuroimaging study. Journal of Interactive Marketing, 47, 150-180.

Chen, Y., Wang, Q. y Xie, J. (2011). Online social interactions: a natural experiment on word of mouth versus observational learning. Journal of marketing research, 48(2), 238-254.

Chevalier, J. y Mayzlin, D. (2006). The effect of word of mouth on sales: online book reviews. Journal of Marketing Research, 43(3), 345-354.

Clauset, A., Shalizi, C. y Newman, M. (2009). Power-law distributions in empirical data. SIAM Review, 51(4), 661-703.

Crawford, G. C., Aguinis, H., Lichtenstein, B., Davidsson, P. y McKelvey, B. (2015). Power law distributions in entrepreneurship: implications for theory and research. Journal of Business Venturing, 30(5), 696-713.

Dellarocas, C. y Narayan, R. (2007). Tall heads vs. long tails: do consumer reviews increase the informational inequality between hit and niche products? School of Business Research Paper, 06-056. 
Elberse, A. (2008). Should you invest in the long tail? Harvard Business Review, 86 (7/8), 88-96.

Elberse, A. y Oberholzer-Gee, F. (2007). Superstars and underdogs: an examination of the long tail phenomenon in video sales. Harvard Business School, 07-015.

Filieri, R., Alguezaui, S. y McLeay, F. (2015). Why do travelers trust TripAdvisor? Antecedents of trust towards consumer-generated media and its influence on recommendation adoption and word of mouth. Tourism Management, 51, 174-185.

Goel, S., Broder, A., Gabrilovich, E. y Pang, B. (2010). Anatomy of the long tail: ordinary people with extraordinary tastes. Proceedings of the Third ACM International Conference on Web Search and Data Mining (pp. 201-210). New York.

Gu, B., Tang, Q. y Whinston, A. (2013). The influence of online word-of-mouth on long tail formation. Decision Support Systems, $56,474-481$.

Hervas-Drane, A. (2009). Word of mouth and taste matching: a theory of the long tail. NET Institute Working Paper, 07-041.

Koçaş, C. y Akkan, C. (2016). A system for pricing the sales distribution from blockbusters to the long tail. Decision Support Systems, 89, 56-65.

Kodinariya, T. y Makwana, P. (2013). Review on determining number of cluster in k-means clustering. International Journal, 1(6), 90-95.

Lee, J., Lee, J. N. y Shin, H. (2011). The long tail or the short tail: the category-specific impact of eWOM on sales distribution. Decision Support Systems, 51(3), 466-479.

Madhulatha, T. (2012). An overview on clustering methods. Journal of Engineering IOSR, 2(4), 719-725.
Mahanti, A., Carlsson, N., Mahanti, A., Arlitt, M. y Williamson, C. (2013). A tale of the tails: power-laws in internet measurements. IEEE Network, 27(1), 59-64.

Manes, E. y Tchetchik, A. (2018). The role of electronic word of mouth in reducing information asymmetry: an empirical investigation of online hotel booking. Journal of Business Research, 85, 185-196.

Martínez-Torres, M. R. (2014). Analysis of open innovation communities from the perspective of social network analysis. Technology Analysis \& Strategic Management, 26(4), 435-451.

Maslowska, E., Malthouse, E. C. y Viswanathan, V. (2017). Do customer reviews drive purchase decisions? The moderating roles of review exposure and price. Decision Support Systems, 98, 1-9.

Moe, W. W. y Trusov, M. (2011). The value of social dynamics in online product ratings forums. Journal of Marketing Research, 48(3), 444-456.

Morales-Arroyo, M. y Pandey, T. (2010). Identification of critical eWOM dimensions for music albums. IEEE International Conference on Management of Innovation and Technology (pp. 1.230-1.235). Singapore.

Newman, M. (2005). Power laws, Pareto distributions and Zipf's law. Contemporary Physics, 46(5), 323-351.

Olmedilla, M. ${ }^{a}$, Martínez-Torres, M. ${ }^{a}$ R. y Toral, S. L. (2015). Examining the power-law distribution among eWOM communities: a characterisation approach of the long tail. Technology Analysis \& Strategic Management, 28(5), 601-613.

Olmedilla, M. ${ }^{a}$, Martínez-Torres, M. ${ }^{a}$ R. y Toral, S. L. (2016). Harvesting big data in social science: a methodological approach for collecting online user-generated content. Computer Standards \& Interfaces, 46, 79-87. 
Park, C. y Lee, T. M. (2009). Information direction, website reputation and eWOM effect: a moderating role of product type. Journal of Business Research, 62(1), 61-67.

Park, D. H., Lee, J. y Han, I. (2007). The effect of on-line consumer reviews on consumer purchasing intention: the moderating role of involvement. International Journal of Electronic Commerce, 11(4), 125-148.

Shao, K. (2012). The effects of controversial reviews on product sales performance: the mediating role of the volume of word of mouth. International Journal of Marketing Studies, 4(4), 32-38.

Standifird, S. (2001). Reputation and ecommerce: eBay auction and the asymmetrical impact of positive and negative ratings. Journal of Management, 27(3), 279-295.

Yeap, J., Ignatius, J. y Ramayah, T. (2014). Determining consumers' most preferred eWOM platform for movie reviews: a fuzzy analytic hierarchy process approach. Computers in Human Behavior, 31, 250-258.

Zhang, L., Ma, B. y Cartwright, D. K. (2013). The impact of online user reviews on cameras sales. European Journal of Marketing, 47(7), 1.115-1.128.

Zhang, K. Z., Zhao, S. J., Cheung, C. M. y Lee, M. K. (2014). Examining the influence of online reviews on consumers' decisionmaking: a heuristic-systematic model. Decision Support Systems, 67, 78-89.

Zhou, W. y Duan, W. (2015). An empirical study of how third-party websites influence the feedback mechanism between online word-of-mouth and retail sales. Decision Support Systems, 76, 14-23.

Zhu, F. y Zhang, X. (2010). Impact of online consumer reviews on sales: the moderating role of product and consumer characteristics. Journal of Marketing, 74(2), 133-148.

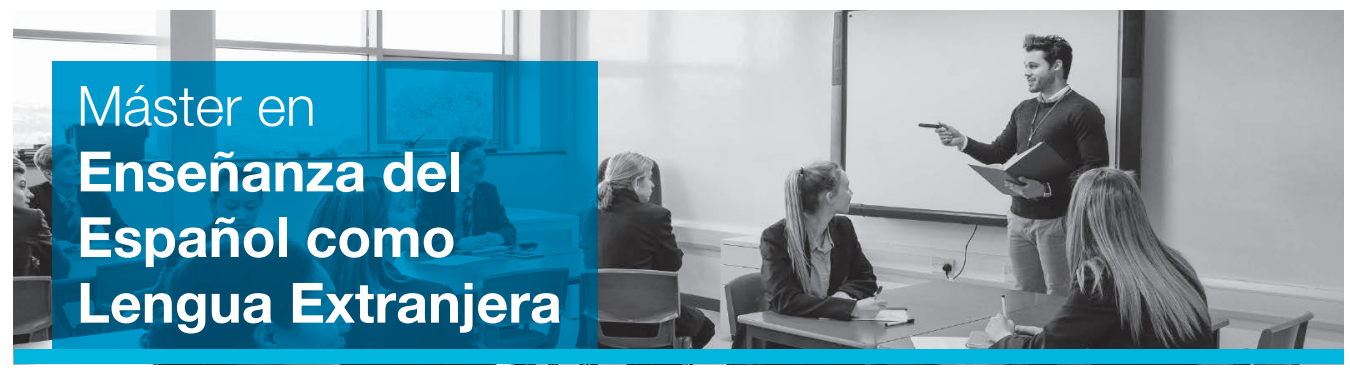

Este máster oficial [60 créditos ECTS] tiene una duración normal de 12 meses.

Dirigido a: Titulados universitarios en las distintas áreas de Lenguas Modernas, Filología, Lingüística, Traducción, Ciencias de la Educación, Humanidades, Pedagogía y Ciencias de la Comunicación y profesionales de las lenguas que deseen especializarse en la enseñanza del español como lengua extranjera.

Objetivos: Formar profesionales cualificados en el ámbito de la enseñanza del español como lengua extranjera, dotándolos de un perfil práctico y competitivo que los capacite para realizar adecuadamente y con garantía de éxito su labor profesional, facilitándoles su integración en un mercado laboral de enorme proyección internacional. 


\section{Magisterio de Educación Infantil}

Hoy en día los centros educativos tienen una imperiosa necesidad de disponer de personal especializado, capaz de hacer frente a las necesidades educativas de la etapa infantil, de acuerdo con los conocimientos ya logrados por las diversas ciencias que hoy se ocupan de los niños en los primeros años de su vida, así como de los logros relativos al desarrollo de la inteligencia, la emocionalidad y la formación de la personalidad temprana, resultantes de estudios recientes sobre el desenvolvimiento de la mente infantil.

\section{Magisterio de Educación Primaria}

Son objetivos de la educación primaria, entre otros: conocer y apreciar los valores y las normas de convivencia, aprender a obrar de acuerdo con ellas, prepararse para el ejercicio activo de la ciudadanía y respetar los derechos humanos, así como el pluralismo propio de una sociedad democrática. También, desarrollar hábitos de trabajo individual y de equipo, de esfuerzo y responsabilidad en el estudio, así como actividades de confianza en uno mismo, sentido crítico, iniciativa personal, curiosidad, interés y creatividad en el aprendizaje.

\section{Menciones en los grados de} Magisterio de Educación Infantil y de Magisterio de Educación Primaria

Mención en Lengua Inglesa

Mención en Pedagogía Terapéutica

Mención en Audición y Lenguaje

Mención en Tecnología Educativa

Mención en Enseñanza de la Religión Católica

\section{Curso de adaptación al grado}

Este curso de adaptación al grado ofrece a los maestros diplomados en la Especialidad de Educación Infantil o Primaria la posibilidad de obtener formación en campos determinados dentro del ejercicio profesional docente en estas etapas, a través de las menciones cualificadoras mencionadas anteriormente.

El objetivo principal del plan de estudios de este curso de adaptación al grado es contribuir a la actualización de la formación de los maestros diplomados. La aplicación de las TIC a la educación y de líneas pedagógicas innovadoras, fruto de la investigación en educación, hacen necesaria la actualización de los conocimientos didácticos de los diplomados y la formación de los maestros en investigación e innovación.

Al finalizar el curso de adaptación se obtiene el título de grado en Magisterio de Educación Infantil o en Magisterio de Educación Primaria.

La docencia en la etapa de educación infantil o primaria es una profesión regulada. Los graduados en Magisterio de Educación Infantil o en Magisterio de Educación Primaria tienen como principal salida profesional el trabajo como profesores en estas etapas, tanto en centros públicos como concertados y privados.

Si bien otras salidas profesionales para estos títulos pueden ser:

- Participación en proyectos educativos de organismos e instituciones (centros culturales, museos, asociaciones, ONG, etc.).

- Centros de educación para adultos.

- Centros de ocio y tiempo libre.

- Participación en programas de extensión educativa (actividades extraescolares, actividades de apoyo, etc.).

- Diseño y elaboración de materiales didácticos.

- Participación en proyectos de atención a la infancia y familiar. 